In this integral, $|T[f(x)]|^{2}$ is an arbitrary positive function of class $L_{1}(-\infty,+\infty)$, and $|T[K(x ; m)]-1|^{2}$ is a positive function, bounded for every $m$. By a theorem of H. Lebesgue, ${ }^{*}$ the conditions (i) and (ii) are necessary and sufficient for the convergence of the above integral to zero, as $m \rightarrow \infty$. This proves the theorem.

Theorem 2. Let condition (ii) of Theorem 1 be replaced by

$$
\lim _{m \rightarrow \infty} \int_{a}^{b}|T[K(x ; m)]|^{2} d x=0,
$$

for every finite interval $(a, b)$. Then (i) and (ii') are necessary and sufficient in order that $\left\|T_{m}(x ; f)-0\right\|_{2}=\left\|T_{m}(x ; f)\right\|_{2} \rightarrow 0$ as $m \rightarrow \infty$.

The method of proof is identical with that of Theorem 1. Both Theorem 1 and Theorem 2 can be extended, mutatis mutandis, to cover the case of any number of dimensions.

YALE UNIVERSITY

\title{
A CORRECTION AND AN ADDITION
}

\section{BY D. N. LEHMER}

In an article in this Bulletin (vol. 39 (1933), p. 764), the total number of squares of order four, magic in the rows and columns, is given as 539,136 , which arise from 468 "normalized" squares. A second, and after that a third, calculation changes this total to 549,504 , which arise from 477 normalized squares. This result is important since it settles definitely in the negative the question as to whether the system of magic squares form a group which is a sub-group of the symmetric group on the number of elements in the square. In fact, the final number contains a factor 53, which is not a factor of the order of the symmetric group on 16 elements. The former erroneous result left this question undecided.

\section{The University of California}

* Annales de la Faculté des Sciences de l'Université de Toulouse, (3), vol. 1, (1909), p. 52. The results of this paper were previously obtained without the use of this theorem of Lebesgue. M. H. Stone kindly pointed out to the author, however, that the work could be considerably shortened by using it. 